\title{
Data-Driven Identification for linear-viscoelastic materials
}

\author{
Gabriel Valdés-Alonzo ${ }^{1, *}$, Adrien Leygue ${ }^{1}$, Christophe Binetruy ${ }^{1}$ and Alberto \\ García-González ${ }^{2}$ \\ ${ }^{1}$ GeM - Research Institute of Civil Engineering and Mechanics \\ École Centrale de Nantes \\ 1 rue de la Noë, 44321 Nantes, France \\ e-mail: gabriel.valdes-alonzo@ec-nantes.fr, adrien.leygue@ec-nantes.fr, \\ christophe.binetruy@ec-nantes.fr \\ ${ }^{2}$ LàCaN -Laboratori de Càlcul Numèric, E.T.S. de Ingeniería de Caminos \\ Universitat Politècnica de Catalunya - BarcelonaTech \\ C/ Jordi Girona 1, E-08034 Barcelona, Spain \\ e-mail: berto.garcia@upc.edu
}

\begin{abstract}
Data-driven Identification (DDI) is a method proposed by Leygue et al. (2018) to estimate the strain-stress relations of materials based on the data-driven computational mechanics paradigm. It relies on a set of strain field measures, which are fed into a minimization problem which solution provides an estimation of mechanical stresses, as well as a database of points that sample the behavior of the material. While this approach works well in elasticity, the dependence of stress on past strain values poses a problem when dealing with viscoelastic materials.

In this work, we account for the time dependence by enriching the existing algorithm with history variables. While the original algorithm defines the state of an element by instantaneous strain and stress, we consider here an extended state with the history of the strain, changing the dimension of the problem: instead of having a strain-stress couple of the form $\left(\epsilon_{t}, \sigma_{t}\right)$, we consider the form $\left(\epsilon_{t}, \ldots, \epsilon_{t-n}, \sigma_{t}\right)$, with $n$ being the amount of previous steps we want to include in the calculation. This methodology was complemented by the use of data analysis techniques, such as kernel Principal Component Analysis (kPCA), which allowed us to improve the estimation of stresses by finding the low dimensional manifold where the data lives. When testing synthetic homogeneous samples for trusses and plane stress, preliminary results indicate that considering past strain values clearly improves the estimation of stresses when compared against the original algorithm, obtaining results where the original DDI would fail.

This new methodology shows promising results, given its improved accuracy. It can also be easily extended to more complex viscoelastic cases given the versatile nature of DDI.
\end{abstract}

\section{ACKNOWLEDGEMENTS}

This project has received funding from the European Union's Horizon 2020 research and innovation programme under the Marie Sklodowska-Curie grant agreement No 764636.

\section{REFERENCES}

[1] Leygue, A. et al. Data-based derivation of material response. Comput. Methods Appl. Mech. Engrg., Vol. 331, pp. 184-196, (2018).

[2] García-González, A. et al. A kernel Principal Component Analysis (kPCA) digest with a new backward mapping (pre-image reconstruction) strategy. arXiv preprint, arXiv:2001.01958. 